\title{
SEQUENTIAL EMITTER IDENTIFICATION METHOD BASED ON D-S EVIDENCE THEORY
}

\author{
Xiaotao Guo $^{1,2}$ - XingWang ${ }^{1}$ - Dongqing Zhou ${ }^{1}$ - Yubing Wang ${ }^{1}$ \\ ${ }^{1}$ Aeronautics and Astronautics Engineering College, Air Force Engineering University, Xi`an 710038, China \\ ${ }^{2}$ School of Electronics and Information Engineering, Xi' an Jiaotong University, Xi an 710049, China
}

\begin{tabular}{l} 
ARTICLE INFO \\
\hline Article history: \\
Received: 13.12 .2015$. \\
Received in revised form: 26.4 .2016$. \\
Accepted: 28.4 .2016$. \\
\hline Keywords: \\
emitter identification \\
D-S evidence theory \\
information fusion \\
bispectra \\
wavelet transforms
\end{tabular}

\section{Introduction}

With the development of the science and technology, "Electromagnetic Dominance" has been the crucial factor of victory in the modern war. From this perspective, study on the location and identification of communication emitters is of great importance, which are the essential prerequisite of the "Electromagnetic Dominance". Many researchers have proposed lots of useful algorithms in this field. Dominique analyzed the RF power amplifier models, which could produce nonlinear distortion that can be applied in the emitter identification [1]. Afterward, a novel method of multi-object optimization system based on high order cumulants is proposed to identify the radio

\begin{abstract}
:
This paper proposes a novel sequential identification method for enhancing the antijamming performance and for accurate recognition rate of the emitters' individual identification in the complicated environment. The proposed method integrates the D-S evidence theory and features extraction that can get the utmost out of features of information systems and decrease the influence of uncertain factors in the signal processing. Firstly, selected features are extracted from intercepted signals. Then, the proposed self-adaptive fusing rule based on the decision vector is utilized to fuse the evidences that are transformed by features and the previous fusing information. Finally, recognition results can be obtained by judgment rules. The simulation analysis demonstrates that self-adaptive fusing rule can achieve a great balance between computational efficiency and accurate identifying rate. While comparing with other identifying methods, the proposed sequential identifying method can provide more accurate and stable recognition results, which makes the utmost care and use of existing information.
\end{abstract}

signal [2]. Besides, many theories are utilized to emitter identification, such as time-frequency analyses [3], fractal feature [4] and selected bispectra [5]. However, these methods cannot achieve the high recognition rate when powerful jamming or other high-density various radio signals exist in complicated electromagnetic environment. Thus, a little more efficient emitter recognition mechanism is required to solve the above identified problem.

The Dempster-Shafer (D-S) evidence theory, an extension and expansion of the probability theory, is an effective method dealing with the uncertain information [6,7]. Aiming at the issues of jamming and environment factors that cause the low accuracy rate of identification, evidence theory could impair 
the influence of these uncertain factors to the final recognition result. Meanwhile, the multi-sensor information fusion could reach higher accurate recognition rate than the single sensor while paying the same price and spending the same time [8]. Although the evidence theory has these advantages, two major issues hinder its extensive application. Acquisition method of the Basic Probability Assignment (BPA) is the first problem. The BPAs used to be obtained mainly via the judgments of experts and previous experience, which is inevitably subjective and seems to lack a theoretical basis. The other issue is the counter-intuitive results generated in the fusing process among highly conflicting evidences.

In this paper, a novel sequential emitter identification method based on self-adaptive evidence fusion is proposed to deal with Special Emitter Identification (SEI) in the complicated electromagnetic environment. The proposed method transforms selected features into corresponding evidences in accordance with the error function between theoretical values and measured values. And the self-adaptive fusing rule can select different fusion method based on the decision vector which is able to judge conflict and consistency degree among evidences. Meanwhile, sequential identification method dilutes the influence of certain identifying error by utilizing previously recognized information. On the basis of above algorithms, the proposed method has higher computing efficiency in the premise of the correctly identified result.

The organization of paper is as follows. In Section 2 , a theoretical method is proposed to transform features into the mass function. Section 3 gives a novel emitter identification method based on evidence theory. In Section 4, illustrative examples are presented to show the practicability of the proposed identifying method. Besides, comparisons between the self-adaptive fusing rule and other methods are made, demonstrating that proposed fusing rule can achieve a great balance between computational efficiency and accurate fusing results. Section 5 summarizes the conclusion of this paper.

\section{Method of evidence acquirement}

The functional block diagram of the emitter identifying the method based on D-S evidence theory is shown in Fig. 1. It is shown that first step of information fusion is how to obtain mass functions of signals. However, there is hardly any method discussing how to obtain the BPAs from the signal features in existing literature about the evidence theory.

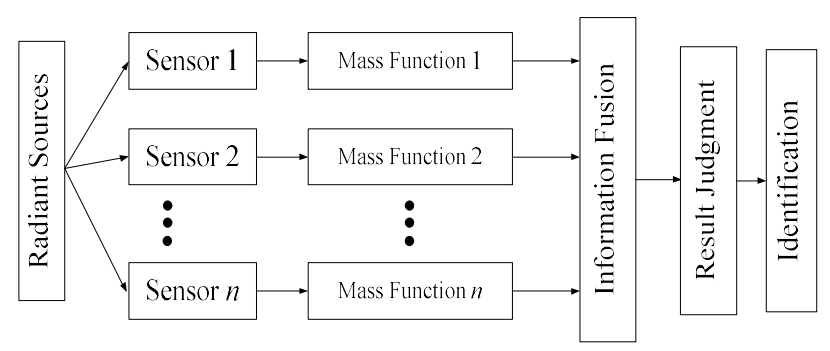

Figure 1. Functional Block Diagram of Emitter Identification

Although some researchers presented the improvement via combing with other theories such as rough set [9] and fuzzy set [10], concrete processes about how to establish the decision tables or subordinating degree function by the intercepted signals are not analyzed. Thus, the method about transforming features into mass functions based on error function is proposed here.

If there are $m$ sensors detecting objective $x$, different sensors can obtain various feature parameters. And the measured data can be regarded as the superposition of the real values and noise. Let observation data from the $i$ th sensor be $x_{i}$ $(i=1,2, \ldots, m), x_{i}$ is subject to Gaussian distribution assuming that the noise is Gaussian white noise with zero mean. So, the probability density function of $x_{i}$ is:

$$
p\left(x / x_{i}\right)=\frac{1}{\sqrt{2 \pi} \sigma} \exp \left(-\frac{(x-\mu)^{2}}{\sigma^{2}}\right)
$$

where $\mu$ is the real value and $\sigma$ is variance of the observation noise.

Assuming that there are $n$ types of known objectives and $m$ sensors, the frame of discernment is $\Omega=\left\{A_{1}\right.$, $\left.A_{2}, \ldots, A_{n}\right\}$, in which $U$ represents the unknown objective. On the basis of the measured data features, error function is

$$
e_{i, A_{j}}(x)=E_{\mathrm{e} r f}\left|\frac{x_{i}-\mu_{i, j}}{\sqrt{2} \sigma_{i}}\right|
$$


where $j=1,2, \ldots, n . \mu_{i, j}$ is real value of the $i$ th objective feature, and $\sigma_{i}$ is noise variance of the $i$ th sensor. Error function $e_{i, A_{j}}(x)$ represents the degree that the observation data of the $i$ th sensor deviates from the objective feature $A_{j}$. Thus, the mass function should be smaller when the error function value is larger, and the metric function is

$$
\begin{gathered}
\alpha_{i, A_{j}}(x)=1-e_{i, A_{j}}(x)=1-E_{\mathrm{e} r f}\left|\frac{x_{i}-\mu_{i, j}}{\sqrt{2} \sigma_{i}}\right| \\
\alpha_{i, U}(x)=1-\operatorname{MAX}\left(\alpha_{i, A_{j}}(x)\right)
\end{gathered}
$$

where $i=1,2, \ldots, m . j=1,2, \ldots, n$. So, the mass function of the sensor evidences is

$$
\begin{aligned}
& m_{i}\left(A_{j}\right)=\frac{\alpha_{i, A_{j}}(x)}{\sum_{j=1}^{n} \alpha_{i, A_{j}}(x)+\alpha_{i, \Omega}(x)} \\
& m_{i}(\Omega)=\frac{\alpha_{i, \Omega}(x)}{\sum_{j=1}^{n} \alpha_{i, A_{j}}(x)+\alpha_{i, \Omega}(x)}
\end{aligned}
$$

Equation (4) meets the characteristics of the mass function and is reserved for the utmost importance of the uncertain information of each sensor. Furthermore, this method avoids the information distortion caused by the subjective mapping.

\section{A novel identification method based on evidence theory}

Given two mass functions $m_{1}$ and $m_{2}$ on $\Omega$, the classical Dempster's fusion rule is

$$
m(A)=\left\{\begin{array}{cl}
0, & A=0 \\
K \sum_{B \cap C=A} m_{1}(B) m_{2}(C), A & \neq 0
\end{array}\right.
$$

where $K=\left[1-\sum_{B \cap C=\varnothing} m_{1}(B) m_{2}(C)\right]^{-1}$ is called the normalization constant. $k=\sum_{B \cap C=\varnothing} m_{1}(B) m_{2}(C)$ reflects the conflict degree among the evidences. It has been proved that errors like counter-intuitive consequences and susceptibility occur when $K_{1}$ is large [11]. To solve these problems, two main methodologies have been extended as follows: the one solution is to pre-process the evidences based on the correlation; the other is to improve the Dempster's fusion rule. The commutative and associative characteristic of the Dempster's fusion rule is one important reason why the evidence theory is widely applied in diverse areas. Although various improved algorithms of the latter idea can convert the conflict section into other focal elements, these algorithms generally destroy the above favorable properties of the fusing rule [12].

The first type of methodology neglects fusion efficiency and condition that there is practically no conflict among the evidences. In this case, Dempster's fusion is superior to other methods dealing with consistent evidences. Thus, selfadaptive evidence fusion based on the modified factor is proposed here, which improves the efficiency of information fusion and provides the excellent quality of the Dempster's fusion rule.

\subsection{Fusion method based on modified factor}

The concept of evidence distance is defined as follows:

$$
d_{i j}=d\left(m_{i}, m_{j}\right)=\sqrt{0.5\left(m_{i}-m_{j}\right)^{T} \mathbf{D}\left(m_{i}-m_{j}\right)}
$$

where $m_{i}$ and $m_{j}$ are two mass functions defined on $\Omega$. $\mathbf{D}$ is a $2^{\mathrm{n}} \times 2^{\mathrm{n}}$ matrix, whose element in $\mathbf{D}$ defined as: $D(A, B)=|A \cap B| /|A \cup B| .|\cdot|$ is cardinality.

It is shown that the less the distances between two BPAs, the more similarities exist between them.

The conflict among evidences is caused by various factors that correlate with different parameters. Just one single parameter cannot reflect the conflict comprehensively. This issue can be illustrated by the following examples.

Example1. Let the frame of discernment be $\Omega=$ $\{\mathrm{A}, \mathrm{B}, \mathrm{C}\}$, and the BPAs are given as

$$
\begin{aligned}
& E_{1}: m_{1}(A)=0.4, m_{1}(B)=0.3, m_{1}(C)=0.3 \\
& E_{2}: m_{2}(A)=0.4, m_{2}(B)=0.3, m_{2}(C)=0.3 \\
& E_{3}: m_{3}(A)=0.4, m_{3}(B)=0.6, m_{3}(C)=0
\end{aligned}
$$

Thus, we obtain

$$
\begin{aligned}
& d_{12}=0, d_{13}=0.3 \\
& k_{12}=0.66, k_{13}=0.66
\end{aligned}
$$


It is concluded that the distance $d_{i j}$ exists differences which reflects the consistency degree between the evidence, while the conflict coefficient $k_{i j}$ is the same. Therefore, judging the conflict among evidences only by the conflict coefficient $k$ cannot reflect the evidence differences on the whole and thus it is not comprehensive.

Example2. Let the mass functions of frame $\Omega=\{A$, $B, C\}$ be given as follows:

$$
\begin{aligned}
& E_{1}: m_{1}(A)=0.5, m_{1}(B)=0.3, m_{1}(C)=0.2 \\
& E_{2}: m_{2}(A)=0.2, m_{2}(B)=0.3, m_{2}(C)=0.5 \\
& E_{3}: m_{3}(A)=0, m_{3}(B)=0.1, m_{3}(C)=0.9
\end{aligned}
$$

Similarly, we have

$$
\begin{aligned}
& d_{12}=0.3, d_{23}=0.35 \\
& k_{12}=0.71, k_{23}=0.52
\end{aligned}
$$

It is shown that the $E_{1}$ and $E_{2}$ two evidence consistencies of objective recognition is higher than that of $E_{2}$ and $E_{3}$ for the reason that $k_{23}$ is smaller. Evidence distance can only reflect the differences of mass functions on the whole and thus judging conflict degree by a single evidence distance $d_{i j}$ is also not comprehensive. Therefore, in order to reflect evidence conflict comprehensively and accurately, the decision vector $V_{d e c}$ is proposed combining conflict coefficient $k$ and evidences distance $d_{i j}$ as $V_{d e c}=[k, d]$. Via setting the threshold values $\alpha$ and $\beta$, evidences can be divided into four sections by $V_{d e c}$.

When $k<\alpha$ and $d<\beta$, evidences are in a less conflict condition and can be fused by Dempster's rule directly which has higher efficiency. And if $k>\alpha$ and $d<\beta$, there exist conflict evidences that should be replaced by the reference evidence $m_{\text {ave }}$ defined as follows:

$$
m_{\text {ave }}=\sum_{i=1}^{n}\left(m_{i} \cdot \frac{\sum_{j=1, j \neq i}^{n} \operatorname{sim}\left(m_{i}, m_{j}\right)}{\sum_{i=1}^{n} \sum_{j=1, j \neq i}^{n} \operatorname{sim}\left(m_{i}, m_{j}\right)}\right)
$$

where the frame of discernment $\Omega$ consists of $n$ independent elements $A_{i}(i=1,2, \ldots, n)$.The similarity coefficient $\operatorname{sim}\left(m_{i}, m_{j}\right)=\frac{m_{i} \bullet m_{j}}{\left(\left\|m_{i}\right\| \cdot\left\|m_{j}\right\|\right)^{1 / 2}}$, where $m_{i} \bullet m_{j}=\sum_{k, l=1}^{2^{n}} m_{i}\left(A_{k}\right) m_{j}\left(A_{l}\right)$. The less the similarity $\operatorname{sim}_{i j}$ is, the less the weight $\mathrm{m}_{\mathrm{i}}$ is when calculating the reference evidence. Thus, reference evidence can be applied in eliminating the conflict among evidences.

When $k<\alpha$ and $d>\beta$, it is concluded that there are evidences differing with others on the whole caused by jamming or other environment factors. For this issue, evidences need to be modified before fusing. Similarly, with the reference evidence, the reliability coefficient is defined as follows:

$$
\operatorname{rel}_{i}=e^{-\sum_{j=1}^{n} d\left(m_{i}, m_{j}\right)}, i=1,2, \cdots, n
$$

The reliability coefficient reflects different levels of evidence research. The larger the reliability coefficient is, the higher the consistency of evidences is on the whole. Beside the reliability coefficient, the uncertain coefficient is defined as:

$$
\text { ucer }_{i}=\frac{A U_{i}}{\max \left\{A U_{i}\right\}}, i=1,2, \cdots, n
$$

where $A U_{i}=-\sum_{\theta_{k}} P_{m_{i}}\left(\theta_{k}\right) \log _{2}\left(P_{m_{i}}\left(\theta_{k}\right)\right) \cdot P_{m}\left(\theta_{k}\right)$ is the pignistic probability function mapped by the BPAs. The pignistic transformation of a belief function $m$ on $\Omega=\left\{A_{1}, A_{2}, \ldots, A_{n}\right\}$ used to be given by

$P_{m_{i}}(A)=\sum_{B \subseteq \Omega} \frac{|A \cap B|}{|B|} \cdot \frac{m_{i}(B)}{1-m_{i}(\varnothing)}, \quad \forall A \subseteq \Omega(10)$

where $|A|$ is the cardinality of set $A$. The essence of this method is to transform the BPAs into probability distribution on the basis of the cardinality of sets. Similar to the maximum entropy, the method makes relatively great amount of information losses. Thus, a novel transformation method is proposed based on the belief function $\mathrm{Bel}$ and plausibility function $\mathrm{Pl}$ of the BPAs as follows [13]: 


$$
\begin{aligned}
P_{m}\left(A_{i}\right)= & \frac{\sum^{B e l} \operatorname{Bel}\left(A_{i}\right)+\left(1-\sum^{B e l}\right) \operatorname{Pl}\left(A_{i}\right)}{\sum_{i}\left(\sum^{B e l} \operatorname{Bel}\left(A_{i}\right)+\left(1-\sum^{B e l}\right) \operatorname{Pl}\left(A_{i}\right)\right)} \\
& \cdot\left(1-\sum^{B e l}\right)+\operatorname{Bel}\left(A_{i}\right)
\end{aligned}
$$

where $\sum^{B e l}$ is Bels' accumulation of the focal elements of the single set. The above method combines the belief function $\mathrm{Bel}$ and plausibility function $P l$ based on the weight of the distributed BPAs value. This method is relatively more reasonable for making the utmost of the given information.

On the basis of the reliability coefficient and uncertainty coefficient, the modified factor of the modified evidence is defined as:

$$
\bmod _{i}=\sqrt{\text { rel }_{i} \bullet \text { ucer }_{i}^{-1}} / \max \left\{\sqrt{\text { rel }_{i} \bullet \text { ucer }_{i}^{-1}}\right\}
$$

Thus, the given evidences could be modified on the foundation of Shafer's reliability rule and the significance of the modified factor if $k<\alpha$ and $d>\beta$, and the modification rule is given as:

$$
m^{\prime}\left(A_{i}\right)=\left\{\begin{array}{lr}
\bmod _{i} \cdot m\left(A_{i}\right), & A_{i} \subseteq \Omega \\
1-\sum_{A_{i} \neq \varnothing} \bmod _{i} \cdot m\left(A_{i}\right), & A_{i}=\Omega
\end{array}\right.
$$

When $k>\alpha$ and $d>\beta$, the conflict evidences should be firstly replaced by the reference evidence $m_{\text {ave }}$ and then the condition whether the recomposed evidences meet inequality $d^{\prime}<\alpha$ or not should be judged. If they do, the recomposed evidences can be fused directly; otherwise, the recomposed evidences would be modified based on the modified factor before fusion.

\subsection{Sequential identification method process based on evidence theory}

On the basis of above theories, sequential identification method based on the D-S evidence theory is proposed here, the process of which is shown in Fig. 2. And the major procedures are given as follows:

Step 1: The appropriate method of feature extraction is selected to process various signals from different emitters, and then, the BPAs are transformed from features by Equation (4). Switch to Step. 2.
Step 2: Judge whether the conflict coefficient $k$ among the evidences is larger than threshold value $\alpha$ or not. If it is, switch to Step 3; otherwise, switch to Step 4.

Step 3: The similarities $\operatorname{sim}_{i j}$ are calculated among the evidences, then the threshold is confirmed by $\lambda=$ $\sigma \sqrt{2 \ln N}$ [14] where $\sigma$ is the standard deviation of similarities $\operatorname{sim}_{i j}$ and $N$ is the number of the evidences. If over half numbers of the similarities $\operatorname{sim}_{i j}$ are less than $\lambda$, the evidence is regarded as the conflict one, and then replaced by the reference evidence $m_{\text {ave }}$ calculated by Equation (7). Switch to Step 4.

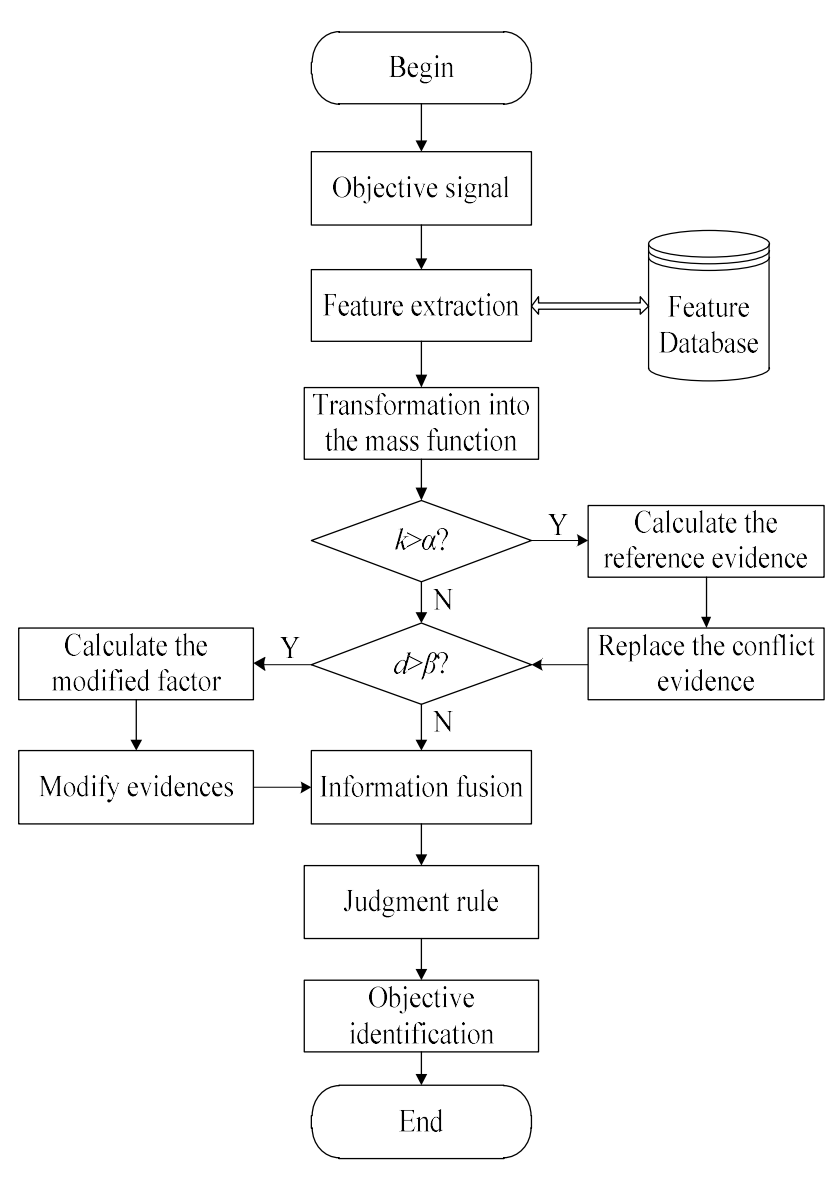

Figure 2. Flow Diagram of Sequential Identification Algorithm

Step 4: Similar to Step 2, each evidence distance $d_{i j}$ among the BPAs should be calculated firstly, and parameter $d$ is the mean value of evidence distances. Judge whether the mean distance $d$ is larger than threshold value $\beta$ or not. If it is, switch to Step 5; otherwise, switch to Step 6. 
Step 5: Calculate the modified factor by Equation (12) and modify the evidences by Equation (13). Switch to Step 6.

Step 6: Fuse the modified evidences by Dempster's rule. Finally, determine the identifying result on the basis of the following three rules.

Assume $\exists A_{1}, A_{2} \subset \Omega$ meet:

$$
\begin{aligned}
& m\left(A_{1}\right)=\max \left\{m\left(A_{i}\right), A_{i} \subset \Omega\right\} \\
& m\left(A_{2}\right)=\max \left\{m\left(A_{i}\right), A_{i} \subset \bar{A}_{1}\right\}
\end{aligned}
$$

There are,

$$
\begin{aligned}
& \text { rule } 1: m\left(A_{1}\right)-m\left(A_{2}\right)>\varepsilon_{1} \\
& \text { rule } 2: m\left(A_{1}\right)>m(\theta) \\
& \text { rule } 3: m(\theta)<\varepsilon_{2}
\end{aligned}
$$

where $\varepsilon_{1}$ and $\varepsilon_{2}$ are pre-set thresholds. When the above three rules are satisfied simultaneously, it can be concluded that the element $A_{1}$ is the identifying result. The values of the parameters $\alpha$ and $\beta$ are selected depending on the practical conditions. The less the values are, the higher the accuracy of identifying consequence is and the slower the convergence process is. Additionally, the greater the values, the higher the risk of selecting the fusing rules.

\section{Experimental investigation}

In order to demonstrate the feasibility and validity of the proposed identifying method, some examples have been carried out. The proposed method is analyzed in two ways: firstly, comparisons are made between self-adaptive fusing rule and other methods; secondly, an application example of the sequential emitter identification method is given.

\subsection{Comparison between self-adaptive fusing rule and other methods}

The simulation data used in Table 1 and Table 2 are quoted from Ref [15]. Consequently, the results derived from different combination rules are listed in Table 3 and Table 4. Original signals are divided into two sections; normal evidences are listed in Table 1 while high conflict evidences are listed in Table 2. In this example, let the $k=0.7$ and the $d=0.5$.
As illustrated in Table 3, when evidences applied to information fusion are in normal condition, Dempster's rule is the most efficient method which provides for the accumulation characteristic of evidence theory. Example results show in Table 3 that all the methods except Yager's and Sun's rule can be convergence to the objective $a$, among which convergence speed of Dempster's rule is the fastest. Convergence of Dempster's rule is evident in two sets of evidences while the one of Pang' exists in four sets. However, by Yager's rule, conflictive parts of evidences are assigned to the uncertain set that is against making judgments. And methods used by Sun and Pang cannot increase the support degree to an objective when the number of evidences increases. Thus, self-adaptive fusing rule can contribute to accumulation characteristics and high convergence speed of Dempster's rule when the decision vector $V_{d e c}$ is less than the threshold, which provides for a balance between computational efficiency and accurate identifying rate.

\section{Table 1. The Normal BPAs Function}

\begin{tabular}{|l|c|c|c|}
\hline \multirow{2}{*}{ Evidences } & \multicolumn{3}{|c|}{ Recognition Framework } \\
\cline { 2 - 4 } & $\mathrm{a}$ & $\mathrm{b}$ & $\mathrm{c}$ \\
\hline $\mathrm{m}_{1}$ & 0.9 & 0 & 0.1 \\
\hline $\mathrm{m}_{2}$ & 0.88 & 0.01 & 0.11 \\
\hline $\mathrm{m}_{3}$ & 0.5 & 0.2 & 0.3 \\
\hline $\mathrm{m}_{4}$ & 0.98 & 0.01 & 0.01 \\
\hline
\end{tabular}

Table 2. The BPAs Function with Conflict Information

\begin{tabular}{|l|c|c|c|}
\hline \multirow{2}{*}{ Evidences } & \multicolumn{3}{|c|}{ Recognition Framework } \\
\cline { 2 - 4 } & $\mathrm{a}$ & $\mathrm{b}$ & $\mathrm{c}$ \\
\hline $\mathrm{m}_{1}$ & 0.9 & 0 & 0.1 \\
\hline $\mathrm{m}_{2}$ & 0 & 0.01 & 0.99 \\
\hline $\mathrm{m}_{3}$ & 0.5 & 0.2 & 0.3 \\
\hline $\mathrm{m}_{4}$ & 0.98 & 0.01 & 0.01 \\
\hline
\end{tabular}

Table 4 shows "One-vote veto" and counterintuitive problems occur when Dempster's rule is used to fuse high conflict evidences. The fusing result cannot be judged by Yager's and Sun's rules because the conflictive part is assigned to the uncertainty set. Meanwhile, convergence speed of Pang's method is too slow to satisfy real time requirement of emitter recognition method. 
Table 3. The Results of the Normal Data

\begin{tabular}{|c|c|c|c|}
\hline \multirow{3}{*}{ Methods } & \multicolumn{3}{|c|}{ Decision-making Results } \\
\cline { 2 - 4 } & Two sets of evidences & Three sets of evidences & Four sets of evidences \\
\hline \multirow{4}{*}{ Dempster } & $\mathrm{m}(\mathrm{a})=0.9863$ & $\mathrm{~m}(\mathrm{a})=0.9917$ & $\mathrm{~m}(\mathrm{a})=0.9999$ \\
& $\mathrm{~m}(\mathrm{~b})=0$ & $\mathrm{~m}(\mathrm{~b})=0$ & $\mathrm{~m}(\mathrm{~b})=0$ \\
& $\mathrm{~m}(\mathrm{c})=0.0137$ & $\mathrm{~m}(\mathrm{c})=0.0083$ & $\mathrm{~m}(\mathrm{c})=0.0001$ \\
& $\mathrm{~m}(\Omega)=0$ & $\mathrm{~m}(\Omega)=0$ & $\mathrm{~m}(\Omega)=0$ \\
\hline \multirow{4}{*}{ Yager[16] } & $\mathrm{m}(\mathrm{a})=0.7920$ & $\mathrm{~m}(\mathrm{a})=0.3960$ & $\mathrm{~m}(\mathrm{a})=0.3881$ \\
& $\mathrm{~m}(\mathrm{~b})=0$ & $\mathrm{~m}(\mathrm{~b})=0$ & $\mathrm{~m}(\mathrm{~b})=0$ \\
& $\mathrm{~m}(\mathrm{c})=0.0110$ & $\mathrm{~m}(\mathrm{c})=0.0033$ & $\mathrm{~m}(\mathrm{c})=0$ \\
& $\mathrm{~m}(\Omega)=0.197$ & $\mathrm{~m}(\Omega)=0.6007$ & $\mathrm{~m}(\Omega)=0.6119$ \\
\hline \multirow{5}{*}{ Sun[17] } & $\mathrm{m}(\mathrm{a})=0.9360$ & $\mathrm{~m}(\mathrm{a})=0.5278$ & $\mathrm{~m}(\mathrm{a})=0.4555$ \\
& $\mathrm{~m}(\mathrm{~b})=0.0008$ & $\mathrm{~m}(\mathrm{~b})=0.0121$ & $\mathrm{~m}(\mathrm{~b})=0.0046$ \\
& $\mathrm{~m}(\mathrm{c})=0.0280$ & $\mathrm{~m}(\mathrm{c})=0.0328$ & $\mathrm{~m}(\mathrm{c})=0.0108$ \\
& $\mathrm{~m}(\Omega)=0.0352$ & $\mathrm{~m}(\Omega)=0.4273$ & $\mathrm{~m}(\Omega)=0.5291$ \\
\hline & $\mathrm{m}(\mathrm{a})=0.9863(\mathrm{a})=0.8493$ & $\mathrm{~m}(\mathrm{a})=0.8829$ \\
& $\mathrm{~m}(\mathrm{~b})=0.0010$ & $\mathrm{~m}(\mathrm{~b})=0.0422$ & $\mathrm{~m}(\mathrm{~b})=0.0339$ \\
& $\mathrm{~m}(\mathrm{c})=0.0137$ & $\mathrm{~m}(\mathrm{c})=0.1085$ & $\mathrm{~m}(\mathrm{c})=0.0833$ \\
& $\mathrm{~m}(\Omega)=0$ & $\mathrm{~m}(\Omega)=0$ & $\mathrm{~m}(\Omega)=0$ \\
\hline \multirow{5}{*}{ This paper } & $\mathrm{m}(\mathrm{a})=0.9863$ & $\mathrm{~m}(\mathrm{a})=0.9917$ & $\mathrm{~m}(\mathrm{a})=0.9863$ \\
& $\mathrm{~m}(\mathrm{~b})=0$ & $\mathrm{~m}(\mathrm{~b})=0$ & $\mathrm{~m}(\mathrm{~b})=0$ \\
& $\mathrm{~m}(\mathrm{c})=0.0137$ & $\mathrm{~m}(\mathrm{c})=0.0083$ & $\mathrm{~m}(\mathrm{c})=0.0137$ \\
& $\mathrm{~m}(\Omega)=0$ & $\mathrm{~m}(\Omega)=0$ & $\mathrm{~m}(\Omega)=0$ \\
\hline
\end{tabular}

Table 4. The Results of the Conflict Data

\begin{tabular}{|c|c|c|c|}
\hline \multirow{3}{*}{ Methods } & \multicolumn{3}{|c|}{ Decision-making Results } \\
\cline { 2 - 4 } & Two sets of evidences & Three sets of evidences & Four sets of evidences \\
\hline \multirow{4}{*}{ Dempster } & $\mathrm{m}(\mathrm{a})=0$ & $\mathrm{~m}(\mathrm{a})=0$ & $\mathrm{~m}(\mathrm{a})=0$ \\
& $\mathrm{~m}(\mathrm{~b})=0$ & $\mathrm{~m}(\mathrm{~b})=0$ & $\mathrm{~m}(\mathrm{~b})=0$ \\
& $\mathrm{~m}(\mathrm{c})=1$ & $\mathrm{~m}(\mathrm{c})=1$ & $\mathrm{~m}(\mathrm{c})=1$ \\
& $\mathrm{~m}(\Omega)=0$ & $\mathrm{~m}(\Omega)=0$ & $\mathrm{~m}(\Omega)=0$ \\
\hline \multirow{4}{*}{ Yager } & $\mathrm{m}(\mathrm{a})=0.7920$ & $\mathrm{~m}(\mathrm{a})=0.3960$ & $\mathrm{~m}(\mathrm{a})=0.3881$ \\
& $\mathrm{~m}(\mathrm{~b})=0$ & $\mathrm{~m}(\mathrm{~b})=0$ & $\mathrm{~m}(\mathrm{~b})=0$ \\
& $\mathrm{~m}(\mathrm{c})=0.1970$ & $\mathrm{~m}(\mathrm{c})=0.0033$ & $\mathrm{~m}(\mathrm{c})=0$ \\
& $\mathrm{~m}(\Omega)=0.1970$ & $\mathrm{~m}(\Omega)=0.6007$ & $\mathrm{~m}(\Omega)=0.6119$ \\
\hline \multirow{4}{*}{ SUN } & $\mathrm{m}(\mathrm{a})=0.1647$ & $\mathrm{~m}(\mathrm{a})=0.0542$ & $\mathrm{~m}(\mathrm{a})=0.0142$ \\
& $\mathrm{~m}(\mathrm{~b})=0.0018$ & $\mathrm{~m}(\mathrm{~b})=0.0081$ & $\mathrm{~m}(\mathrm{~b})=0.0013$ \\
& $\mathrm{~m}(\mathrm{c})=0.2984$ & $\mathrm{~m}(\mathrm{c})=0.0836$ & $\mathrm{~m}(\mathrm{c})=0.0087$ \\
& $\mathrm{~m}(\Omega)=0.5350$ & $\mathrm{~m}(\Omega)=0.8541$ & $\mathrm{~m}(\Omega)=0.9780$ \\
\hline \multirow{5}{*}{ Pang } & $\mathrm{m}(\mathrm{a})=0.4055)=0.5251$ & $\mathrm{~m}(\mathrm{a})=0.7065$ \\
& $\mathrm{~m}(\mathrm{~b})=0.0045$ & $\mathrm{~m}(\mathrm{~b})=0.0936$ & $\mathrm{~m}(\mathrm{~b})=0.0697$ \\
& $\mathrm{~m}(\mathrm{c})=0.5900$ & $\mathrm{~m}(\mathrm{c})=0.3813$ & $\mathrm{~m}(\mathrm{c})=0.2238$ \\
& $\mathrm{~m}(\Omega)=0$ & $\mathrm{~m}(\Omega)=0$ & $\mathrm{~m}(\Omega)=0$ \\
\hline & $\mathrm{m}(\mathrm{a})=0.6837$ & $\mathrm{~m}(\mathrm{a})=0.6861$ & $\mathrm{~m}(\mathrm{a})=0.9941$ \\
& $\mathrm{~m}(\mathrm{~b})=0.0114$ & $\mathrm{~m}(\mathrm{~b})=0.0238$ & $\mathrm{~m}(\mathrm{~b})=0.0025$ \\
& $\mathrm{~m}(\mathrm{c})=0.0848$ & $\mathrm{~m}(\mathrm{c})=0.0964$ & $\mathrm{~m}(\mathrm{c})=0.0033$ \\
& $\mathrm{~m}(\Omega)=0.2201$ & $\mathrm{~m}(\Omega)=0.1937$ & $\mathrm{~m}(\Omega)=0.0001$ \\
\hline
\end{tabular}


In contrast to above algorithms, the proposed method can replace the conflict evidences by reference evidence, through which the conflict degree of evidences decreases. It can also strengthen the weight of credible evidence while weakening the weight of incredible evidence when decision vector $V_{d e c}$ is larger than the threshold. Furthermore, the self-adaptive fusing method can reach the convergence rapidly and avoid the counter-intuitive results.

\subsection{Proposed method's application in emitter identification}

In order to achieve the identification, the features used to emitter recognition must have time-invariant feature, scale transform feature and phase stability feature [12]. Thus, this paper has selected wavelet analysis and selected bispectra to extracting features in target signal. Comparison between a single emitter recognition method and the proposed one is analyzed as follows.

\subsubsection{Features Extraction Based on the Wavelet Analysis}

The discrete wavelet transform (DWT) of a signal $s(t)$ is defined as follows:

$$
C(j, k)=\sum_{n \in Z} s(n) \psi_{j, k}(n)
$$

where $s(n)$ is the analyzed signal. $\psi_{j, k}(n)$ is wavelet function, in which $j$ is scale coefficient and $k$ is translation coefficient.

The signal $s(n)$ is decomposed into high-frequency and low-frequency two parts by wavelet analysis, in which approximation coefficients comprise the original signal features and the detail coefficients include the subtle information of the analyzed signal. Then, the next scale DWT is applied to decomposing approximation coefficients. Supposed that the scale of DWT is $n, n$ detail coefficients and 1 approximation coefficient can be obtained finally.

The feature vector $\boldsymbol{F}=\left\{F_{i}\right\}$ can be gained by calculating the power of two types of coefficients, which is defined as follows:

$$
F_{i}=\left[\frac{1}{K} \sum_{k=1}^{K} W_{i k}\right]^{1 / 2}
$$

where $K$ and $W$ are the numbers of wavelet coefficients and the $k$ th wavelet coefficient when

the scale of decomposition is $i . n$ is the decomposition level of wavelet analysis. Thus, the feature vector $\boldsymbol{F}$ represents the detail feature information of radiating signals.

\subsubsection{Extracting Features Based on the Selected Bispectra}

For the discrete signal $x(t)$ whose mean value is zero, the bispectra is:

$$
B\left(\omega_{1}, \omega_{2}\right)=\sum_{\tau_{1}=-\infty}^{+\infty} \sum_{\tau_{2}=-\infty}^{+\infty} C_{3 x}\left(\tau_{1}, \tau_{2}\right) e^{-j\left(\omega_{1} \tau_{1}+\omega_{2} \tau_{2}\right)}
$$

$$
\text { where } \begin{aligned}
C_{3 x}\left(\tau_{1}, \tau_{2}\right) & =\sum_{t=-\infty}^{+\infty} x^{*}(t) x\left(t+\tau_{1}\right) x\left(t+\tau_{2}\right) \\
& =E\left\{x^{*}(t) x\left(t+\tau_{1}\right) x\left(t+\tau_{2}\right)\right\}
\end{aligned} .
$$

For the convenience of description, let $\omega=\left(\omega_{1}, \omega_{2}\right)$ and $B(\omega)=B\left(\omega_{1}, \omega_{2}\right)$. Assuming that $\left\{B_{k}^{(i)}(\omega)\right\}_{k=1,2, \cdots, N_{i}}$ and $\left\{B_{k}^{(j)}(\omega)\right\}_{k=1,2, \cdots, N_{j}}$ are the bispectra assemblages via calculating where the subscript $k$ represents the bispectra from the $k$ th group of observation data and the superscripts $i, j$ are type of the signals, the definition of the Fisher information separability measure [14] is:

$$
m^{(i, j)}(\omega)=\frac{\sum_{l=i, j}\left[\operatorname{mean}_{k}\left(B_{k}^{(l)}(\omega)\right)-\operatorname{mean}_{l}\left[\operatorname{mean}_{k}\left(B_{k}^{(l)}(\omega)\right)\right]\right]^{2}}{\sum_{l=i, j} \operatorname{var}\left(B_{k}^{(l)}(\omega)\right)}
$$

where $\operatorname{mean}_{k}\left(B_{k}^{(l)}(\omega)\right)$ and $\operatorname{var}\left(B_{k}^{(l)}(\omega)\right)$ represent mean value and variance of the $l$ th group of signals' bispectra at frequency $\omega=\left(\omega_{1}, \omega_{2}\right)$. mean $_{l}\left[\operatorname{mean}_{k}\left(B_{k}^{(l)}(\omega)\right)\right]$ represents the population 
central mean of the all type signals bispectra at frequency $\omega$.

It is represented that separability measure between the $i$ th and the $j$ th two group of signals is larger if the $m^{(i, j)}(\omega)$ is larger. The $L$ frequency value having largest separability measure could be selected to group into the assemblage as the characteristic frequency, and the central frequency is the characteristic frequency in double frequency surface. On the basis of the above concepts, the selected bispectra is combined by the characteristic frequency and the central frequency.

\subsubsection{Comparison simulation}

In a multi-sensor target recognition system, there are totally three types of targets: $\Omega=\{A, B, C\}$. Assuming that the real objective is the $A$ and SNR is $0 \mathrm{~dB}$, the signal wave is shown in Fig. 3. Meanwhile, the detail feature information extracted by wavelet analysis is shown in Fig. 4 by setting $n=7$. Feature is selected on the basis of the selected bispectra shown in Fig. 5. The BPAs based on two algorithms are obtained by Equation (11). Table 5 shows the mass function transformed from features and previous fusing information. The $\mathrm{m}_{1}$ and $\mathrm{m}_{2}$ two mass functions are transformed by wavelet and selected bispectra two methods, respectively, and the $\mathrm{m}_{3}$ has the origin from previous information.

Table 5 shows that the objective cannot be confirmed only with a single feature because the BPAs among these methods don't satisfy identification judgment rule. The BPAs between two objectives may be too close to recognize the target acquired by the single sensor. This issue is due to great low SNR caused by powerful jamming and complicated electromagnetic environment.

In real condition, data reconnoitered by different sensors ordinarily are not in high conflict while each BPA cannot support the judgment enough. Thus, the proposed method can identify the real objective via multi-sensor information fusion that makes the full use of obtained information. Even if there are conflict evidences caused by some sensors error, the proposed method can decrease the conflict degree among evidences by replacing conflict evidences with reference evidence. In this way, robustness and stability of the proposed method are increased. At the same time, support degree to real objective can rise by evidence fusion. The above mentioned analysis illustrates that performance of the proposed method is more excellent than the one of the existing method.

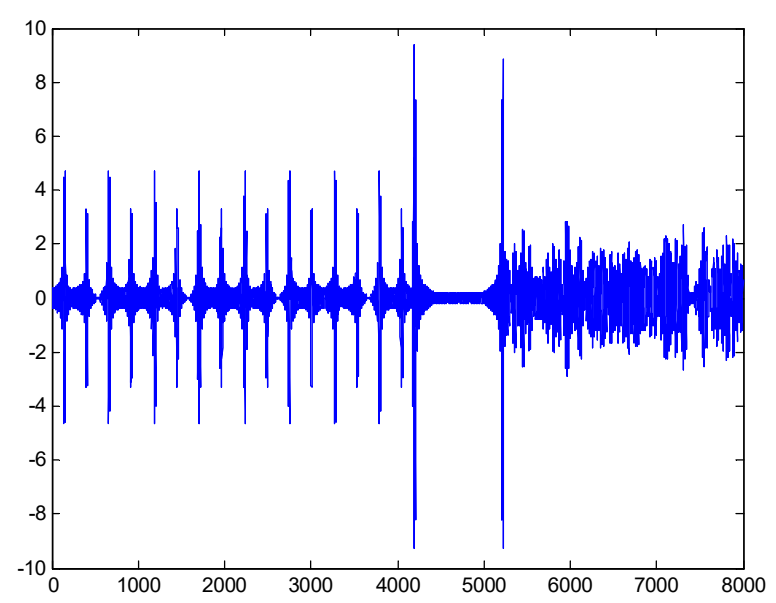

Figure 3. The Emitter Signal Wave

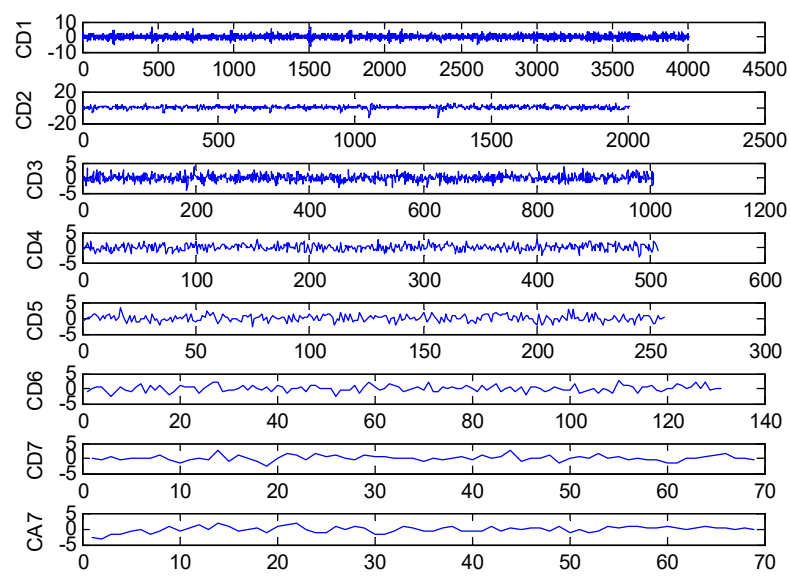

Figure 4. Detail Feature Information Extracted by Wavelet Analysis

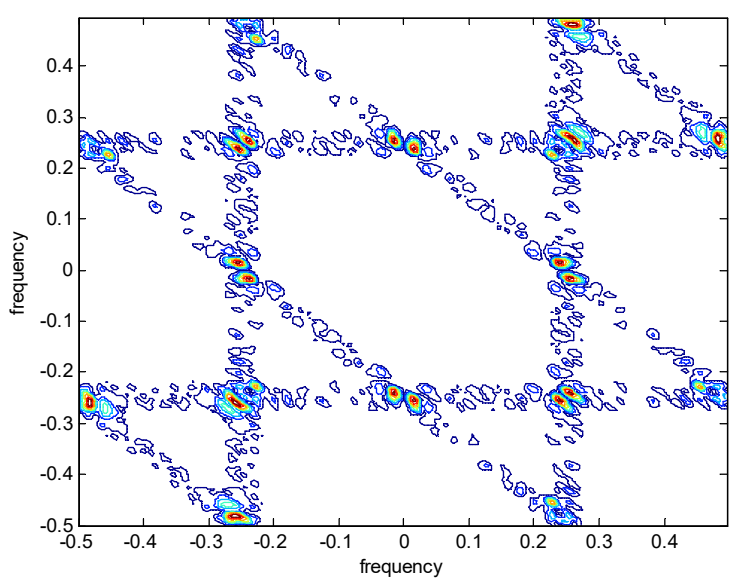

Figure 5. Features Extracted by Bispectra 
Table 5. The BPAs Function of the Target Recognition System

\begin{tabular}{|c|c|c|c|c|c|}
\hline \multirow{2}{*}{ Evidences } & \multicolumn{4}{|c|}{ Recognition Framework } & \multirow{2}{*}{ Recognition Results } \\
\cline { 2 - 5 } & $\mathrm{A}$ & $\mathrm{B}$ & $\mathrm{C}$ & $\Omega$ & \\
\hline $\mathrm{m}_{1}$ & 0.4127 & 0.2643 & 0.1512 & 0.1718 & uncertain \\
\hline $\mathrm{m}_{2}$ & 0.6202 & 0.3094 & 0.0704 & 0 & uncertain \\
\hline $\mathrm{m}_{3}$ & 0.5301 & 0.2103 & 0.2514 & 0.0082 & uncertain \\
\hline $\mathrm{m}$ & 0.8465 & 0.1279 & 0.0256 & 0 & $\mathrm{~A}$ \\
\hline
\end{tabular}

\section{Conclusion}

In this paper, a novel sequential method of emitter recognition based on the improved D-S evidence theory is proposed to deal with individual identification in complicated electromagnetic environment. The proposed method can be divided into two categories, one is to extract signal features and to transform them into the BPAs, and the other is to fuse the evidences on the basis of self-adaptive fusion rule. From analysis and the results of simulation, the following conclusions could be drawn:

1) Compared with other fusing methods based on D$\mathrm{S}$ evidence theory, the proposed method of transforming signal features into the BPAs is more practical and more systematic, which avoids subjectivity of expert judgments. Meanwhile, the proposed fusing rule can arrive at an optimal trade-off between its fusing accuracy and computational efficiency.

2) The proposed sequential recognition method could improve the accuracy and stability of the emitter identification results by multi-sensors information fusion and making the full use of the previous information.

3) Compared with other identifying methods, the improved method can reduce effects of the jamming and environment factors to its utmost when identifying each time.

\section{Acknowledgements}

This work is supported by the Aviation Science Foundations named "Validity of Airborne Sensors Information Detection and Management Technology" (20145596025) and "Key Technologies and Experimental Method of Evaluation on Airborne LPI Radar" (20152096019). The authors are also grateful to Dr. Wenzhe Wang for his help in collecting the data.

\section{References}

[1] Dominique, S., Mairtin, O., Anthony, A. G., Michael, G.: RF power amplifier behavioral modeling, Cambridge University Press, Cambridge, 2009.

[2] Tang, Z. L., Yang, X. N., Li J. D.: Identification of Radio Communication Source with Multi-object Optimization, Journal of Applied Sciences, 30 (2012), 6, 559-565.

[3] Avdakovic, S., Bosovic, A., Hasanspahic, N., et al.: Time-frequency analyses of disturbances in power distribution systems, Engineering Review, 34 (2014), 3, 175-180.

[4] Tang, Z. L., Yang, X.N., Li, J. D.: Study on Fractal Features of Modulated Radio Signal, Chinese Journal of Physics, 60 (2011), 5, 401407.

[5] Xu, S. H., Huang, B. X., Huang, Y. C., et al.: Identification of individual radio transmitter based on selected surrounding-line integral bispectra, Proceedings of the 9th International Conference on Advanced Communication Technology, Phoenix Park, 2007,1147-1150.

[6] Li, Z. Z., Wu, F.B., Wang, W.: A clustering algorithm based on D-S evidence theory for wireless sensor networks, Information Technology Journal, 13 (2014), 13, 2211-2217.

[7] Huang, C. X., Bao, W. X.: A remote sensing image fusion algorithm based on the second generation curvelet transform and DS evidence theory, J Indian Soc Remotr Sens, 42 (2014), 3,645-650.

[8] Jiang, Y., Xiao, J.: Target tracking based on a multi-sensor covariance intersection fusion Kalman filter, Engineering Review, 34 (2014), 1, 47-54.

[9] Han, L., Shi, L. P.: Approach to evidence combination based on rough set, 2009 International Conference on Electronic Computer Technology, Macau, 2009, 693-697. 
[10] Xu,Y. K., Liang, X. G., Jia, X. H.: Information fusion based on fuzzy evidence theory and its application in target recognition, Journal of Harbin Institute of Technology, 44 (2012), 3,107-111.

[11] Lefevre, E., Colot, O., Vannoorenberghe, P.: How to preserve the conflict as an alarm in the combination of belief function, Decis Support Syst, 2013, 56, 326-333.

[12] Yamada, K.: A new combination of evidence based on compromise, Fuzzy Sets and Systems, 159 (2008), 13, 1689-1708.

[13] Jiang, W., Wu, C. C., Jia, J., et al.: $A$ probabilistic transformation of basic pro bability assignment in $D$-S evidence theory, Journal of Northwestern Polytechnical University, 31 (2013), 2, 295-299.
[14] Donoho, D. L., Johnstone, I. M.: Ideal spatial adaptation by wavelet shrinkage, Biometrika, 81 (1994), 3, 425-455.

[15] Wei, Y.C.: A novel D-S combination method of conflicting evidences based on Pearson correlation coefficient, Telecommunication Engineering, 52 (2012), 4, 466-471.

[16] Yager, R. R.: On the Dempster-Shafer framework and new combination rules, Information System, 41 (1989), 2, 93-137.

[17] Sun, Q., Ye, X.Q., Gu, W. K.: A new combination rules of evidence theory, Acta Electronica Sinica, 28 (2000), 8, 117-119.

[18] Pang, J. F., Lin, Y., Li, Y. B., et al. A new DS evidence fusion algorithm based on cosine similarity coefficient, International Conference on Measurement, Information and Control, Harbin, 2013, 1487-1490. 